\title{
Neuropsychiatric aspects of Huntington's disease
}

\author{
J S Paulsen, R E Ready, J M Hamilton, M S Mega, J L Cummings
}

Department of

Psychiatry, The University of Iowa, 2880 JPP, 200 Hawkins

Drive, Iowa City, IA

52242, USA

J S Paulsen

\section{Department of}

Neurology

J S Paulsen

Department of

Psychology

J S Paulsen

R E Ready

Department of

Psychiatry, UCSD

School of Medicine,

San Diego, CA, USA

J M Hamilton

Department of

Psychology, SDSU,

San Diego, CA, USA

J M Hamilton

Department of

Neurology, UCLA

School of Medicine,

Los Angeles, CA, USA

M S Mega

J L Cummings

Department of Psychiatry

J L Cummings

Department of Biobehavioral Sciences

J L Cummings

Correspondence to: Dr J S Paulsen

jane-paulsen@uiowa.edu

Received 12 June 2000 and in revised form

30 January 2001

Accepted 12 March 2001

\begin{abstract}
Objective-Neuropsychiatric symptoms are common in Huntington's disease and have been considered its presenting manifestation. Research characterising these symptoms in Huntington's disease is variable, however, encumbered by limitations within and across studies. Gaining a better understanding of neuropsychiatric symptoms is essential, as these symptoms have implications for disease management, prognosis, and quality of life for patients and caregivers.
\end{abstract}

Method-Fifty two patients with Huntington's disease were administered standardised measures of cognition, psychiatric symptoms, and motor abnormalities. Patient caregivers were administered the neuropsychiatric inventory.

Results-Ninety eight per cent of the patients exhibited neuropsychiatric symptoms, the most prevalent being dysphoria, agitation, irritability, apathy, and anxiety. Symptoms ranged from mild to severe and were unrelated to dementia and chorea.

Conclusions-Neuropsychiatric symptoms are prevalent in Huntington's disease and are relatively independent of cognitive and motor aspects of the disease. Hypothesised links between neuropsychiatric symptoms of Huntington's disease and frontal-striatal circuitry were explored. Findings indicate that dimensional measures of neuropsychiatric symptoms are essential to capture the full range of pathology in Huntington's disease and are vital to include in a comprehensive assessment of the disease.

(F Neurol Neurosurg Psychiatry 2001;71:310-314)

Keywords: Huntington's disease; neuropsychiatry; dementia

Huntington's disease is characterised by a triad of symptoms including motor disturbance, cognitive impairment, and psychiatric features. Although it is well established that psychiatric symptoms are common in Huntington's disease $^{1-5}$ research and clinical emphases have been on motor and cognitive aspects of the disorder. Reconsideration of the psychiatric manifestations of the disease is warranted. Firstly, psychiatric disturbances are most strongly associated with stress, disability, and placement decisions. ${ }^{2}{ }^{6}$ Secondly, there is evidence that brain changes precede the traditional clinical diagnosis and psychiatric manifestations may predate motor signs by a decade. ${ }^{78}$ Finally, clinical trials are underway to slow Huntington's disease progression in clinically affected patients with plans to treat "preclinical" patients imminent. Careful study of neuropsychiatric symptoms associated with Huntington's disease is essential to help distinguish features that are pathognomonic from behaviours that are sensitive but not specific of the disease.

Models emphasising the role of the basal ganglia in neuropsychiatric disorders have been described over the past several decades. ${ }^{9-11}$ One recent overview suggests that because the earliest pathological changes in Huntington's disease are in the associative portions of the striatum, the behaviours first affected by disease may be cognitive and psychiatric, rather than motor. ${ }^{12}{ }^{13}$ Indeed, reviews of psychiatric symptoms in Huntington's disease have suggested that depression, apathy, aggression, and disinhibition are common and suicide rates are over four times those of the general population. ${ }^{14}$ Previous studies have used an array of data collection methods, however, and standardised uniform assessment of psychiatric features in Huntington's disease is lacking. The purpose of the current study is to use the well established neuropsychiatric inventory $\left(\mathrm{NPI}^{15}\right)$ to better characterise psychiatric symptoms in the disease.

\section{Methods}

SAMPLE

Participants were 52 consecutive patients with Huntington's disease recruited from the Huntington's Disease Clinical Research Program at the University of California at San Diego $(n=29)$ and from the University of Iowa Huntington's Disease Society of America Center of Excellence $(n=23)$. The diagnosis was made by a senior staff neurologist based on the presence of an unequivocal movement disorder in a person with a family history of Huntington's disease. All diagnoses were confirmed by DNA analysis for CAG expansion in the IT 15 region of chromosome $4 .^{16}$ All consecutive persons with an unequivocal diagnosis attending the UCSD Huntington's disease clinic between 1994 and 1997 or attending the University of Iowa Huntington's disease clinic between 1997 and 1999 were approached for inclusion in the study. No patient refused participation in the study. Four patients were excluded from the study for the following reasons: (a) having a medical illness other than Huntington's disease $(n=1)$; (b) having a history of head trauma with loss of consciousness $(n=1)$; and (c) having an age of onset younger than $18(n=2)$. Patients were $52 \%$ women with a mean age of 45.5 (SD 11.7) years (range 26-78) and a mean of 13.3 (SD 2.3) (range 6-17) years of education. Duration of disease (number of years since diagnosis) ranged from 0 to 20 years (mean 4.7 (SD 4.4)). Age of onset of illness ranged from 
25 to 57 with a mean of 43.1 (SD 9.6). There were no juvenile onset cases included in this study. Mini mental state examination ${ }^{17}$ (MMSE) scores ranged from 7 to 30 (mean 24.34 (SD 4.38), and Mattis dementia rating scale $^{18}$ (DRS) scores ranged from 63 to 142 (mean 122 (SD 15.6)). Total chorea scores (assessed with the unified Huntington's disease rating scale ${ }^{19}$ (UHDRS) ranged from 0 to 26 (mean 12.4 (SD 7.4)). Over $79 \%$ of patients were taking no medication $(n=41)$. Four patients were taking a selective seratonin reuptake inhibitor (SSRI) and a typical neuroleptic drug, although dosages of the neuroleptic drug were very low in every case $(<5$ $\mathrm{mg})$. One patient was taking an atypical neuroleptic drug. Four patients were taking SSRIs only and one patient was taking trazodone at bedtime for sleep. No patients were taking tetrabenazine. Analyses conducted with and without the subsample of patients taking psychotropic medications did not differ.

\section{PROCEDURE}

Huntington's disease caregivers $(n=52)$ were interviewed with the neuropsychiatric inventory $^{15}$ (NPI) by JSP or a trained research assistant. The NPI assesses the frequency (four point rating scale) and severity (three point scale) of 10 neuropsychiatric disturbances (delusions, hallucinations, agitation, dysphoria, anxiety, euphoria, apathy, disinhibition, irritability, aberrant motor behaviour), and a score from 0 to 12 was obtained for each scale by multiplying frequency by severity. An NPI total score was calculated by summing ratings for all symptoms. The NPI offers advantages over previous psychiatric research in Huntington's disease; it is standardised, is not influenced by normal aging, it is used to characterise various neurological disorders, ${ }^{20-22}$ and it is used as a measure of treatment response $\mathrm{e}^{23}$ and in investigations of the biological correlates of neuropsychiatric symptoms. Informant reports of psychiatric symptoms in patients with neurodegenerative disease are commonly used and have been found to agree significantly with self reports. ${ }^{24}$

Patients were administered the DRS and MMSE cognitive screening tests and the UHDRS as a measure of chorea severity. Scores on the MMSE were unavailable for two patients. After complete description of the study to the patients and caregivers as approved by the institutional review boards of both universities, informed consent was obtained.

Neuropsychiatric symptoms were not normally distributed. Therefore, all analyses used non-parametric tests. Specifically, Spearman correlation coefficients were used to examine associations between factors, and MannWhitney $U$ tests were used for all comparisons of means. Due to the many analyses, a significance level of 0.01 was selected to control for multiple comparisons and to guard against type I errors.
Table 1 Descriptons of NPI neuropsychiatric symptoms in Huntington's disease

\begin{tabular}{llll}
\hline NPI Scale & Frequency & Meant & $S D$ \\
\hline Dysphoria & 69.2 & 3.12 & 3.46 \\
Agitation & 67.3 & 2.88 & 3.32 \\
Irritability & 65.4 & 2.63 & 3.11 \\
Apathy & 55.8 & 2.79 & 4.02 \\
Anxiety & 51.9 & 1.96 & 3.14 \\
Disinhibition & 34.6 & 1.29 & 2.77 \\
Euphoria & 30.8 & 1.04 & 2.27 \\
Delusions & 11.5 & 0.75 & 2.63 \\
Aberrant motor & 9.6 & 0.60 & 2.18 \\
Hallucinations & 1.9 & 0.23 & 1.66 \\
\hline
\end{tabular}

$\mathrm{n}=52$.

NPI=Neuropsychiatric inventory.

$\star$ Per cent of patients with HD with an NPI scale score $\geqslant 1$.

$\dagger$ Mean scores are averages of frequency $\times$ severity ratings.

\section{Results}

NEUROPSYCHIATRIC SYMPTOM PREVALENCE

Only one patient did not exhibit any neuropsychiatric symptoms. The most often endorsed symptoms were dysphoria $(69.2 \%)$, agitation $(67.3 \%)$, irritability $(65.4 \%)$, apathy $(55.8 \%)$, and anxiety (51.9\%). These symptoms also had the highest mean scale scores, which reflect frequency and severity ratings. Delusions (11.5\%), aberrant motor behaviour (9.6\%), and hallucinations $(1.9 \%)$ were the least prevalent and had the lowest frequency by severity mean ratings (table 1 ).

ASSOCIATIONS OF NPI WITH DEMOGRAPHIC AND DISEASE MEASURES

The total NPI score was negatively correlated with years of education $(r=-0.37)$. MannWhitney $U$ tests comparing symptom means in male and female patients showed no significant differences. Age was negatively correlated with disinhibition $(r=-0.41)$ and delusions $(r=-0.36)$. Illness duration and chorea severity were not significantly associated with any NPI scores (table 2).

Neither measure of dementia severity (MMSE, DRS) was significantly correlated with neuropsychiatric symptoms (table 2). The DRS initiation and perseveration subscale showed a significant positive correlation with delusions $(r=0.36)$. DRS attention was positively correlated with NPI aberrant motor behaviour $(r=0.37)$ and dysphoria $(r=0.41)$. DRS conceptualisation was positively correlated with aberrant motor behaviour $(r=0.37)$. DRS supermarket verbal fluency was positively correlated with delusions $(r=0.38)$.

\section{NEUROPSYCHIATRIC SYMPTOM}

INTERCORRELATIONS

Intercorrelations with the NPI scale were investigated to assess associations among symptoms and to determine if symptom associations found in other neuropsychiatric populations were replicated. Nine significant symptom associations were found (table 3 ). The high correlation $(r=0.81)$ between irritability and agitation suggests that these two scales are essentially measuring the same construct in this sample of patients with Huntington's disease.

Three of the significant NPI symptom associations (agitation and irritability, anxiety and dysphoria, disinhibition, and irritability) were 
Table 2 Correlations between NPI neuropsychiatric symptoms and motor and cognitive measures for patients with HD

\begin{tabular}{|c|c|c|c|c|c|c|c|c|}
\hline \multirow[b]{2}{*}{ NPI Scale } & \multirow[b]{2}{*}{ Chorea } & \multirow[b]{2}{*}{ MMSE† } & \multirow[b]{2}{*}{$D R S \ddagger$} & \multicolumn{5}{|c|}{ DRS Subscales } \\
\hline & & & & Attention & Conceptualisation & Construction & $\begin{array}{l}\text { Initiation and } \\
\text { perseveration }\end{array}$ & Memory \\
\hline Agitation & -0.02 & -0.09 & -0.11 & -0.15 & -0.13 & 0.02 & -0.10 & -0.01 \\
\hline Dysphoria & -0.18 & 0.11 & 0.30 & $0.41^{\star \star}$ & 0.25 & -0.21 & 0.29 & -0.05 \\
\hline Irritability & -0.14 & -0.09 & -0.08 & -0.23 & -0.18 & -0.08 & 0.01 & -0.00 \\
\hline Anxiety & 0.11 & 0.06 & 0.09 & 0.13 & 0.11 & -0.01 & 0.06 & -0.03 \\
\hline Apathy & -0.12 & -0.15 & -0.07 & 0.01 & -0.12 & -0.16 & 0.04 & -0.17 \\
\hline Euphoria & 0.17 & -0.08 & 0.06 & -0.07 & -0.07 & 0.09 & 0.09 & 0.10 \\
\hline Disinhibition & 0.05 & 0.01 & -0.10 & -0.24 & -0.23 & -0.09 & 0.04 & 0.04 \\
\hline Delusions & -0.23 & 0.24 & 0.22 & 0.06 & -0.07 & 0.09 & $0.36^{\star \star}$ & 0.22 \\
\hline Aberrant motor & 0.12 & 0.09 & 0.24 & $0.37^{\star \star}$ & $0.37^{\star \star \star}$ & -0.15 & 0.18 & -0.01 \\
\hline Hallucinations & -0.12 & 0.21 & 0.15 & 0.15 & 0.09 & 0.11 & 0.20 & -0.12 \\
\hline NPI Total & -0.03 & 0.01 & 0.04 & 0.03 & -0.03 & -0.12 & 0.11 & -0.02 \\
\hline
\end{tabular}

NPI=Neuropsychiatric inventory; DRS=Mattis dementia rating scale; MMSE=mini mental state examination; HD=Huntington's disease.

$\nmid \mathrm{n}=50 ; \ddagger \mathrm{n}=52$.

$\star \star \mathrm{p}<0.01$.

Table 3 NPI neuropsychiatric symptom correlations in Huntington's disease

\begin{tabular}{llllllllll}
\hline NPI Scale & Agitation & Dysphoria & Irritability & Anxiety & Apathy & Euphoria & Disinhibition & Delusions & Aberrant Motor \\
\hline Dysphoria & 0.26 & & & & & & & & \\
Irritability & $0.81^{\star}$ & 0.20 & & & & & & \\
Anxiety & $0.41^{\star}$ & $0.40^{\star}$ & $0.43^{\star}$ & & & & & \\
Apathy & 0.09 & 0.14 & 0.14 & -0.05 & & & & \\
Euphoria & 0.31 & 0.10 & 0.25 & 0.25 & -0.08 & & & \\
Disinhibition & $0.38^{\star}$ & 0.22 & $0.37^{\star}$ & 0.34 & 0.08 & 0.30 & & & \\
Delusions & 0.08 & 0.17 & 0.21 & 0.27 & 0.09 & 0.02 & $0.40^{\star}$ & & \\
Aberrant motor & -0.01 & 0.31 & 0.07 & 0.28 & 0.34 & -0.05 & 0.05 & 0.12 & $0.47^{\star}$ \\
Hallucinations & -0.17 & 0.23 & 0.13 & 0.24 & 0.23 & -0.09 & 0.24 & $0.42^{\star}$ & 0.24
\end{tabular}

$\mathrm{n}=52$.

NPI=Neuropsychiatric inventory.

${ }^{\star} \mathrm{p}<0.01$.

also significant $(\mathrm{p}<0.01)$ in an Alzheimer's disease $(n=50)$ sample. ${ }^{22}$ Two significant associations from this sample (apathy and aberrant motor behaviour, dysphoria, and irritability) were not replicated. A significant association between anxiety and agitation found in a sample $(n=22)$ of patients with progressive supranuclear palsy ${ }^{20}$ was replicated in the Huntington's disease sample.

\section{Discussion}

Neuropsychiatric symptoms were common in this Huntington's disease sample, with $98 \%$ of patients exhibiting at least one symptom during the previous month. This total incidence is higher than that found in other dementias ${ }^{22}$ and greater than previously reported in patients with other basal ganglia disorders ${ }^{21}$ also using the NPI. Findings are consistent, however, with other estimates of psychiatric symptoms in Huntington's disease..$^{1-4}$

Dysphoria, agitation, irritability, apathy, or anxiety were present in over $50 \%$ of the sample, whereas psychotic symptoms (delusions and hallucinations) and aberrant motor behaviour were rare. Findings from this investigation are consistent with and enlarge past research on neuropsychiatric aspects of Huntington's disease. Specifically, previous findings indicating that affective disorders ${ }^{1}$ and symptoms ${ }^{4}$ are common in Huntington's disease were replicated. Apathy and irritability have similarly been found to be evident in about half of patients with Huntington's disease. ${ }^{25}$

Given the input and output nuclei of the basal ganglia, it is not surprising that rates of neuropsychiatric symptoms are consistently high in Huntington's disease. The dorsolateralsubcortical circuit projects from dorsal, anterior, and lateral regions of the prefrontal cortex via the dorsolateral head of the caudate, to the ventral anterior and medial dorsal nuclei of the thalamus, and plays a part in complex cognitive processes including working memory, generative behaviours, and the ability to establish and shift from one topic to another. Impairments in these so called "executive functions" also affect various aspects of emotional and behavioural control. For instance, regulatory functions of the circuit (generating and shifting) may alter the appropriate expression of frustration, resulting in apparent irritability and agitation.

The orbitofrontal circuit projects from the anterior and lateral orbitofrontal cortex via the ventromedial caudate nucleus to the ventral anterior and medical dorsal nuclei of the thalamus, and has been associated with disorders including depression and obsessive-compulsive disorder. Most clinical and research reports have found that in virtually all cases, the manifestation of depression was either simultaneous with or after the motor onset of Huntington's disease, suggesting that depression in Huntington's disease is likely due to the disease process. Given that most patients in the current study were prescribed antidepressant medications, it is likely that rates of depression in Huntington's disease are much higher than that reported in the literature.

The medial prefrontal circuit projects from the paralimbic cortical territories (posteromedial orbitofrontal cortex and anterior cingulate) via the nucleus accumbens to the medial dorsal nucleus within the thalamus, and plays a 
part in response inhibition. Dysfunction of the medial circuit produces apathy, which has been shown to increase with illness duration in Huntington's disease. ${ }^{12627}$ These findings are consistent with the known degeneration in the disease, progressing from medial to lateral and from dorsal to ventral caudate, possibly impacting on the orbital and dorsolateral circuits before the cingulate circuit. Although apathy was noted in over $50 \%$ of patients with Huntington's disease, Litvan et $a l^{21}$ showed that patients with hypokinetic movement disorders were more likely to demonstrate hypoactive behaviours, such as apathy, whereas patients with hyperkinetic movement disorders (for example, Huntington's disease) demonstrated relatively more hyperactive behaviours (agitation, irritability, and anxiety). This behavioural dissociation is consistent with circuitry involvement, suggesting hypoactive behaviours in persons with cingulate disruption and hyperactive behaviours in people with orbito/dorsal dysfunction.

Associations found between neuropsychiatric symptoms and demographic factors suggested that younger patients were more likely to experience psychotic symptoms and to be disinhibited. Although this finding is consistent with anecdotal reports, the pathophysiology of this association (age and psychosis) is unknown. It is possible that onset of Huntington's disease at an earlier age disrupts frontostriatal circuitry that is not fully developed, resulting in variations in phenotype. Alternatively, other mediating factors likely exist to account for the variability in age of Huntington's disease onset. It is possible that genetic factors influencing age at onset also impact on behavioural phenotype so that mechanisms influencing earlier onset also increase the probability of psychosis. Given that CAG repeat length accounts for only half of the variance in age of onset, ${ }^{28}$ additional research is needed to better understand Huntington's disease phenotype and onset heterogeneity.

The increased breadth and dimensional assessment of neuropsychiatric symptoms provided by this study showed that patients with Huntington's disease exhibited the full range of symptomatology, from mild to severe, as indicated by NPI frequency and severity ratings. Thus, assessment tools that rely on categorical diagnostic criteria to assess psychiatric aspects of Huntington's disease would likely fail to detect the significant proportion of patients with subthreshold symptoms. Dimensional assessment tools, such as the NPI, may aid in the detection of mild or atypical cases of neuropsychiatric disorders and allow the capture of a full range of symptomatology. Neuropsychiatric assessment at varying degrees of severity also is important because these symptoms can be targeted for intervention and assessment can be used to track change over time during the natural course of the disease, or used to measure response to treatment.

Consistent with previous reports ${ }^{25}$ few associations were found between neuropsychiatric symptoms and measures of cognitive or motor signs. Specifically, the MMSE, total DRS, chorea severity, and disease duration were not significantly correlated with any neuropsychiatric symptom. These findings are consistent with other research ${ }^{21}$ and indicate that behavioural aspects of basal ganglia diseases are often independent of cognitive and motor symptoms, at least early in the disease.

Findings from this study have important implications for clinical neuropsychiatry. The current study suggests that nearly all patients with Huntington's disease experience psychiatric symptoms. In addition, recent research as well as anecdotal evidence implies that psychiatric symptoms may be the first manifestation of disease in up to $79 \%$ of patients. ${ }^{5}$ It is likely, then, that clinical neuropsychiatrists may be among the professionals first and most often seen by persons with Huntington's disease. Unfortunately, specific training for behavioural management of patients with Huntington's disease is not typically available in general psychiatry and neurology programmes. Results of this study suggest that the infrequent patient with Huntington's disease should be considered an opportunity for training and that psychiatric assessment should be a standard component of the Huntington's disease evaluation. Excellent references for treatment can be found in Rosenblatt et $a t^{29}$ and Leroi and Michalon. $^{30}$

This study was supported by National Institutes of Mental Health (NIMH) grants (5R29MH55331-05 and $1 \mathrm{~K} 02 \mathrm{MH} 01579-01)$, a National Institutes of Neurological Disorders and Stroke grant (PO410951-G), a Howard Hughes Medical Institute Pilot Grant, and a Carver Medical Research Initiative Grant Award to JSP, a National Institute on Aging (NIA) Alzheimer's Disease Research Center grant, an Alzheimer's Disease Research Center of California grant, and the Sidell-Kagan Foundation to JLC; a NIA grant to MSM the Sidell-Kagan Foundation to JLC; a NIA grant to MSM
(K08 AG100784); a NIMH grant (R03MH59430-01) to JMH; and a NIH/NIA (T32AG00214) fellowship to RER.

1 Caine ED, Shoulson I. Psychiatric syndromes in Huntington's disease. Am F Psychiatry 1983;140:728-33.

2 Cummings JL. Behavioral and psychiatric symptoms associated with Huntington's disease. In: Weiner WJ, Lang AE, eds. Behavioral neurology of movements disorders. New York: Raven Press, 1995:179-86.

3 Folstein SE, Folstein MF. Psychiatric features of Huntington's disease: recent approaches and findings [review]. Psychiatric Developments 1983;1:193-205.

4 Shiwach R. Psychopathology in Huntington's disease patients. Acta Psychiatr Scand 1994;90:241-6.

5 Morris M. Psychiatric aspects of Huntington's disease: In: Morris M. Psychiatric aspects of Huntington's disease: In:
Harper PS, ed. Huntington's disease. London: WB SaunHarper PS, ed. Huntingt

6 Dewhurst K, Oliver JE, McKnight AL. Socio-psychiatric consequences of Huntington's disease. $\mathrm{Br} \mathcal{F}$ Psychiatry 1970;116:255-8.

7 Gutekunst C, Li S, Yi H, et al. Nuclear and neuropil aggregates in Huntington's disease: relationship to neuropathology. F Neurosci 1999;19:2522-34.

8 Cha JH, Kosinski CM, Kerner JA, et al. Altered brain neurotransmitter receptors in transgenic mice expressing a portion of an abnormal human Huntington disease gene. Proc Natl Acad Sci USA 1998;95:6480-5.

9 Nauta WJ, Mehler WR. Projections of the lentiform nucleus in the monkey. Brain Res 1966;1:3-42.

10 Swerdlow NR, Koob GF. Lesions of the dorsomedial nucleus of the thalamus, medial prefrontal cortex and pedunculopontine nucleus: effects on locomotor activity mediated by nucleus accumbens-ventral pallidal circuitry. Brain Res 1987;412:233-43.

11 Cummings JL. Frontal-subcortical circuits and human behavior. Neurological Review 1993;50:873-80.

12 Vonsattel JP, Difiglia M. Huntington's disease [review]. f Neuropathol Exp Neurol 1998; 57:369-84.

13 Middleton FA, Strick PL. Basal ganglia and cerebellar loops: motor and cognitive circuits. Brain Res Rev 2000;31: 236-50.

14 Di Maio L, Squitieri F, Napolitano G, et al. Suicide risk in Huntington's disease. F Med Genet 1993;30:293-5. 
15 Cummings JL, Mega M, Gray K, et al. The neuropsychiatric inventory: comprehensive assessment of psychopatholric inventory: comprehensive assessment of p
ogy in dementia. Neurology 1994;44:2308-14.

16 The Huntington's Disease Collaborative Research Group. A novel gene containing a trinucleotide repeat that is expanded and unstable on Huntington's disease chromosomes. Cell 1993;72:971-83.

17 Folstein MF, Folstein SE, McHugh PR. Mini-mental state. A practical method for grading the cognitive state of patients for the clinician. F Psychiatr Res 1975;12:189-98.

18 Mattis S. Mental status examination for organic mental syndrome in the elderly patient. In: Bellak L, Karasu TB, eds. Geriatric psychiatry: New York, Grune and Stratton,1976: 77-122.

19 The Huntington Study Group. Unified Huntington's disease rating scale: reliability and consistency. Mov Disord 1996;11:136-42.

20 Litvan I, Mega MS, Cummings JL, et al. Neuropsychiatric aspects of progressive supranuclear palsy. Neurology 1996 ; aspects of pro $1184-9$.

21 Litvan I, Paulsen JS, Mega MS, et al. Neuropsychiatric assessment of patients with hyperkinetic and hypokinetic assessment of patients with hyperkinetic and hypolin

22 Levy ML, Miller BL, Cummings JL, et al. Alzheimer disease and frontotemporal dementias. Behavioral distinctions. Arch Neurol 1996;53:687-90.
23 Kaufer D. Beyond the cholingeric hypothesis: the effect of metrifonate and other cholinesterase inhibitors on neuropsychiatric symptoms in Alzheimer's disease. Dement Geriatr Cogn Disord 1998;2:8-14.

24 Riso LP, Klein DN, Anderson RL, et al. Concordance between patients and informants on the personality disorder examination. Am f Psychiatry 1994;151:568-73.

25 Paulsen JS, Stout JC, Delapena J, et al. Frontal behavioral syndromes in cortical and subcortical dementia. Assessment 1996;3:327-37.

26 Paulsen JS, Butters N, Sadek JR, et al. Distinct cognitive profiles of cortical and subcortical dementia in advanced illness. Neurology 1995;45:951-6.

27 Levy ML, Cummings JL, Fairbanks LA, et al. Apathy is not depression. I Neuropsychiatry Clin Neurosci 1998;10:31419.

28 Andrew SE, Goldberg YP, Hayden MR. Rethinking genotype and phenotype correlations in polyglutamine expansion disorders. Hum Mol Genet 1997;6:2005-10.

29 Rosenblatt A, Ranen NG, Nance MA, et al. A physician's guide to the management of Huntington's disease. guide to the management of Huntington's

30 Leroi I, Michalon M. Treatment of the psychiatric manifestations of Huntington's disease: a review of the literature [review]. Can F Psychiatry 1998;43:933-40.

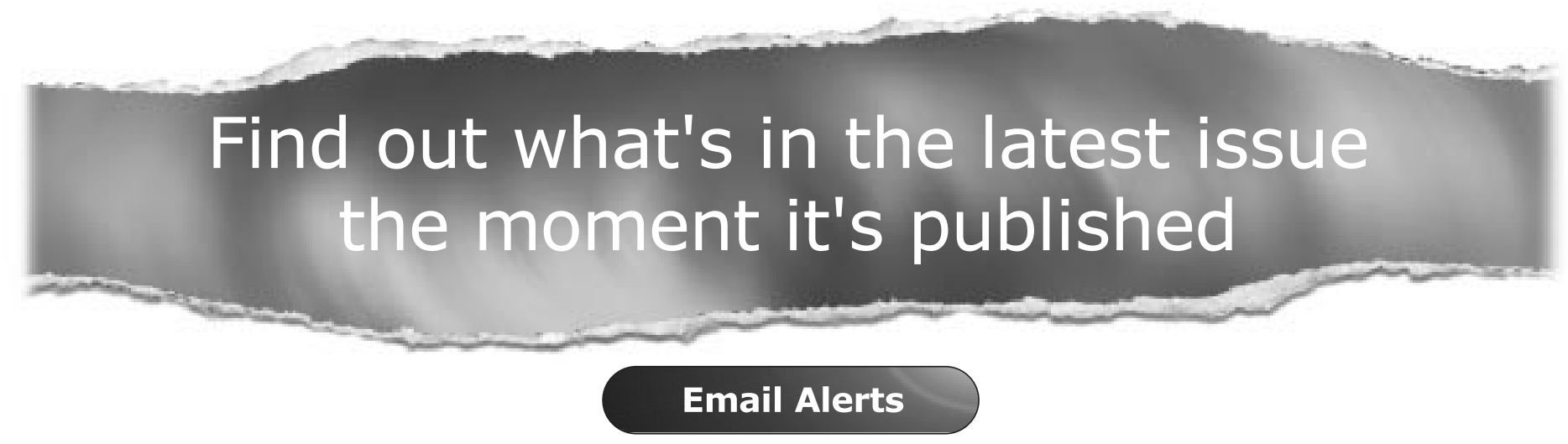

Sign up to receive the table of contents by email every month. You can select from three alerts: Table of Contents (full), TOC Awareness (notice only); Journal of Neurology, Neurosurgery, and Psychiatry related announcements.

\section{www.jnnp.com}

